\title{
Etats-Unis : le e-learning dans l'enseignement supérieur
}

Catherine Bouët

\section{OpenEdition}

12 Journals

Édition électronique

URL : https://journals.openedition.org/ries/1786

DOI : 10.4000/ries. 1786

ISSN : 2261-4265

Éditeur

France Education international

\section{Édition imprimée}

Date de publication : 1 avril 2002

Pagination : 16-18

ISBN : 2-84520-553-8

ISSN : $1254-4590$

Référence électronique

Catherine Bouët, «Etats-Unis : le e-learning dans l'enseignement supérieur », Revue internationale d'éducation de Sèvres [En ligne], 29 | avril 2002, mis en ligne le 01 avril 2005, consulté le 06 juillet 2021. URL : http://journals.openedition.org/ries/1786 ; DOI : https://doi.org/10.4000/ries.1786

Ce document a été généré automatiquement le 6 juillet 2021

(C) Tous droits réservés 


\title{
Etats-Unis : le e-learning dans l'enseignement supérieur
}

\author{
Catherine Bouët
}

1 Les États-Unis sont les créateurs et les premiers utilisateurs du e-learning; ils détiennent $70 \%$ du marché mondial de ce nouveau mode d'apprentissage, qui présente de multiples avantages mais également des contraintes et bouleverse le modèle traditionnel de l'enseignement.

2 On recense aux États-Unis cinq millions d'étudiants qui ont déjà suivi un cours en ligne donnant droit à des unités de valeur. Le nombre d'apprenants qui étudie à la maison croît de façon exponentielle : il a plus que doublé entre 1994 et $1999^{1}$. D'après le Centre national des statistiques de l'éducation (NCES), plus de 1,6 million d'étudiants étaient inscrits à des cours à distance en 1997-98 ${ }^{2}$. Une seconde enquête ${ }^{3}$ fait apparaître que sur 39 États interrogés, les $2 / 3$ ont instauré une université virtuelle ou un système de communauté virtuelle qui exploite le e-learning. Plusieurs types d'activités émergent : les universités virtuelles, les bibliothèques virtuelles telles celle du Wisconsin system qui propose des collections en ligne, la formation continue. Les universités se regroupent et développent de plus en plus de partenariats, dépassant ainsi les concurrences habituelles. Les partenariats public-privé en e-learning sont eux aussi de plus en plus fréquents. Au Colorado par exemple, certaines entreprises accordent des donations aux programmes scolaires et aux institutions éducatives. Les États investissent également dans la formation de personnes qualifiées pour répondre aux besoins suscités par le $e$ learning. Une enquête révèle par ailleurs que la population américaine est à $82 \%$ favorable aux technologies éducatives dans l'enseignement ${ }^{4}$. Au niveau national, un important programme avait été lancé en 1998 par le ministère de l'Éducation nationale fédéral. Ce programme, intitulé Learning Anytime Anywhere Partnerships (LAAP), avait pour objectif d'octroyer des fonds aux institutions qui développaient des programmes d'e-learning. Ainsi le fonds fédéral finance aujourd'hui jusqu'à $50 \%$ des subventions accordées ${ }^{5}$.

3 Les raisons du succès du e-learning sont multiples. Ce mode d'apprentissage permet un gain de temps, puisqu'il abolit les contraintes physiques, et un gain d'argent par 
rapport à l'éducation traditionnelle (le e-learning est environ 15 à $20 \%$ moins cher). Le e-learning permet de personnaliser l'apprentissage. De plus, le Conseil de la Recherche nationale estime que l'interactivité peut aider l'apprenant à mieux visualiser, comprendre les concepts difficiles et à s'auto-évaluer. Enfin, les contenus des cours et les matériels peuvent être facilement et instantanément actualisés. Selon la NASBE, si les technologies de l'information et de la communication sont utilisées de manière idoine, elles peuvent améliorer l'accès à la formation, le contenu et la méthode de l'apprentissage.

4 La pratique du e-learning aux États-Unis ne se fait cependant pas sans contraintes. L'accès au e-learning nécessite un équipement spécifique. En effet, les universités n'ont pas toutes les moyens de se procurer l'accès à l'Internet à haut débit. Se pose également la question de l'accès au réseau informatique en dehors des heures de cours. Les disparités entre les universités ne risquent-elles pas d'être exacerbées ${ }^{6}$ ?

5 Les contenus du e-learning sont également l'objet de quelques critiques. Certains éducateurs pensent que le e-learning peut être inefficace pour certaines matières et chez les étudiants pour qui l'oral et le visuel favorisent l'apprentissage. La fédération américaine des enseignants, (AFT), remarque en outre que de sérieux problèmes surviennent quand le e-learning est conçu sur le modèle des entreprises. En effet, le public ciblé devient un consommateur et les produits sont dès lors standardisés. Les investisseurs privés envahissent le marché et leur offre de logiciels et de ressources en ligne est d'une qualité très variable ${ }^{7}$. Par conséquent, la fédération américaine des enseignants (AFT) préconise que les professionnels de l'enseignement décident des contenus pédagogiques.

6 De plus, le e-learning connaît un taux d'abandon plus important que l'enseignement traditionnel. L'appauvrissement du lien entre l'élève et l'enseignant, la difficulté de travailler en équipe et l'appréhension peu concrète des situations sont autant de facteurs de démotivation pour les étudiants. Ainsi, la plupart des éducateurs qui pratiquent le e-learning restent persuadés que ce dernier bénéficie davantage aux étudiants matures et très motivés qui possèdent de fortes aptitudes à la communication écrite. Le e-learning seul est insuffisant s'il n'est pas accompagné d'autres ressources en ligne telles que les bibliothèques ou les messageries permettant de dialoguer avec des spécialistes. Enfin, les éducateurs réclament à l'instance éducative gouvernementale de leur État un conseil pour utiliser les outils et améliorer l'instruction. La demande d'une prise en main du e-learning par l'État fédéral demeure également très forte.

7 Ces réflexions montrent que le e-learning n'est pas seulement une affaire de technologie. L'université transformée par le e-learning doit en effet accompagner cette mutation d'autres réformes telles que la révision des méthodes pédagogiques, le développement des systèmes d'évaluation informatisés et le regroupement de services techniques auparavant dispersés.

8 Le Sénat américain a voté en novembre 2001 un article qui sonne le glas du programme LAAP. Désormais la création de programmes de e-learning par les universités est rendue plus difficile. 


\section{NOTES}

1. National Association of State Boards of Education (NASBE), Any time, any place, any path, any pace, 2001. Document électronique consulté le 5 décembre 2001 [http://www.nasbe.org/ e_learning.html].

2. Distance education: guidelines for good practice, Programme pour l'enseignement supérieur et Conseil politique de la fédération américaine des enseignants, rapport de mai 2000, p. 5. Document électronique consulté le 5 décembre 2001 [http://www.aft.org/higher_ed/ downloadable/distance.pdf]

3. National Governors Association (NGA) Center for Best Practices, The State of E-Learning in the States, 2001, p.10. Document électronique consulté en novembre 2001 [http:// www.educause.edu/asp/doclib/abstract.asp ?ID =CSD1597].

4. NASBE, id., p. 8.

5. U.S. Department of Education, Learning Anytime Anywhere Partnerships, 2001. Document électronique consulté le 20 novembre 2001. [http://www.ed.goov/offices/OPE/FIPSE/LAAP/FY2001/ intro. html].

6. Le rapport de la NASBE note à ce sujet que les universités comprenant une majorité d'étudiants pauvres ont moins de possibilités d'accès à l'éducation en ligne.

7. Selon un chercheur, $90 \%$ des ressources en ligne sont de mauvaise qualité. Jane M. Healy citée dans Any time, any place, any path, any pace, NASBE, 2001, p. 8.

INDEX

Index géographique : États-Unis

\section{AUTEUR}

\section{CATHERINE BOUËT}

Documentaliste au Centre de ressources documentaires du CIEP 